\title{
SIFAT ASIMTOTIK ESTIMATOR NADARAYA-WATSON DENGAN KERNEL ORDE TAK HINGGA
}

\author{
Maria Suci Apriani ${ }^{a}$, Sri Haryatmi ${ }^{\text {b }}$ \\ ${ }^{a}$ Program Studi Pendidikan Matematika FKIP USD \\ Kampus 3 Paingan, Yogyakarta 55282, maria.suci@usd.ac.id \\ burusan Matematika FMIPA UGM \\ Sekip Utara Yogyakarta, s_kartiko@yahoo.com
}

\begin{abstract}
ABSTRAK
Artikel ini menjelaskan sifat kenormalan dan kekonsistenan dari estimator Nadaraya Watson dengan menggunakan kernel berorde tak hingga secara asimtotik. Penelitian ini menggunakan metode studi literatur berdasarkan artikel berjudul Minimally Biased Nonparametric Regression and Autoregression yang dibahas oleh Timothy dan Dimitris. Hasil penelitian ini menunjukkan bahwa estimator Nadaraya Watson dengan menggunakan kernel orde tak hingga memiliki sifat normal dan konsisten secara asimtotik.
\end{abstract}

Kata kunci: Regresi nonparametrik, transformasi Fourier, deret Taylor

\begin{abstract}
This article investigated the normality and consistency of Nadaraya Watson estimator with infinite order kernel asymptotically. This research used literature study method based on the article entitle "Minimally Biased Nonparametric Regression and Autoregression" by Timothy and Dimitris. The result showed that Nadaraya Watson estimator with infinite order kernel has normal and consistent characteristics asymptotically.
\end{abstract}

Key words: Nonparametric regression, Fourier transformation, Taylor series

\section{Pendahuluan}

Mengestimasi fungsi regresi $r\left(X_{i}\right)$ pada model regresi $Y_{i}=r\left(X_{i}\right)+\varepsilon_{i}$ dengan pendekatan nonparametrik, dilakukan jika tidak ada asumsi tentang fungsi $r\left(X_{i}\right)$. Salah satu teknik yang dapat digunakan untuk mengestimasi fungsi tersebut dengan pendekatan nonparametrik adalah teknik penghalusan dengan kernel. Kernel berdasarkan ordenya dibedakan menjadi kernel berorde hingga dan tak hingga. Menurut Timothy dan Dimitris (2003) jika kernel $K$ mempunyai orde $v$ dan fungsi kepadatan $r$ mempunyai turunan kontinu sebanyak $k$ kali maka:

$$
\operatorname{Bias}(\hat{r}(x))=\mathrm{C}_{K, r}(x) h^{n}+o\left(h^{n}\right) .
$$

Ketika fungsi $r$ cukup mulus atau dapat dideferensialkan sebanyak $k$ kali dimana $v \geq k$, maka bias $\hat{r}(x)$ dapat direduksi menjadi $o\left(h^{k}\right)$ dengan secara tepat memilih kernel dengan orde yang lebih besar dari banyaknya diferensial. Namun kita kesulitan untuk menentukan orde kernel berapakah yang harus dipilih. Sehingga ditetapkan suatu kernel yang 
memiliki "infinite orde". Sifat secara asimtotik dari estimator Nadaraya Watson dengan kernel orde tak hingga yaitu:

1. memiliki sifat konsisten secara probabilitas

2. berdistribusi normal dengan rata-rata

$$
\text { nol dan variansi } \frac{\int K^{2}(s) d s \sigma^{2}(x)}{f(x)} \text {, }
$$

akan dibahas lebih dalam pada artikel ini.

\section{Metode Penelitian}

Metodologi yang digunakan dalam penelitian ini adalah studi literatur. Langkah-langkah yang dilakukan penulis adalah sebagai berikut:

1. Mencari dan menentukan jurnal yang akan dijadikan bahan acuan.

2. Mengumpulkan jurnal-jurnal lain yang relevan dengan materi dalam jurnal acuan.

3. Mempelajari buku-buku pendukung yang berkaitan dengan topik permasalahan penelitian.

4. Mempelajari dan membahas topik penelitian yang meliputi: teori regresi nonparametrik, ide dasar smoothing, estimator kernel, estimasi fungsi dalam regresi nonparametrik, sifatsifat fungsi kernel, estimasi densitas kernel, fungsi estimator Nadaraya Watson, kernel dengan infinite orde.
5. Mempelajari performance (bias dan variansi) dari pembilang dan penyebut estimator NadarayaWatson dengan infinite orde kernel.

6. Menyusun laporan penelitian.

\section{Hasil dan Pembahasan}

Hasil yang diperoleh menunjukkan bahwa estimator Nadaraya Watson dengan Kernel orde tak hingga memiliki sifat konsisten secara probabilitas dan normal secara asimtotik. Berikut pembahasannya:

\section{Estimator Nadaraya Watson}

Estimator fungsi regresi yang diusulkan oleh Nadaraya-Watson untuk fungsi densitas $f$ yang tidak diketahui adalah:

$$
\hat{r}(x)=\frac{\frac{1}{n} \sum_{i=1}^{n} K_{h}\left(x-X_{i}\right) Y_{i}}{\frac{1}{n} \sum_{k=1}^{n} K_{h}\left(x-X_{k}\right)}=\frac{\hat{g}(x)}{\hat{f}(x)} .
$$

Menurut Hardle (1991) nilai-nilai statistik pembilang dari estimator Nadaraya-Watson dengan fungsi kernelnya mempunyai orde dua adalah sebagai berikut:

$$
\begin{aligned}
\operatorname{Bias}(\hat{g}(x))= & \frac{h^{2}}{2} g "(x) \mu_{2}(K)+o\left(h^{2}\right), \quad h \rightarrow 0 \\
\operatorname{var}(\hat{g}(x))= & (n h)^{-1} f(x) s^{2}(x)\|K\|_{2}^{2}+ \\
& o\left((n h)^{-1}\right), \text { untuk } n h \rightarrow \infty
\end{aligned}
$$




$$
\begin{aligned}
\operatorname{MSE}(\hat{g}(x))= & (n h)^{-1} f(x) s^{2}\|K\|_{2}^{2}+ \\
& \frac{h^{4}}{4}\left(g "(x) \mu_{2}(K)\right)^{2}+ \\
& o((n h))^{-1}+o\left(h^{4}\right), \\
& h \rightarrow 0, n h \rightarrow \infty
\end{aligned}
$$

dengan $s^{2}(x)=E\left[Y^{2} \mid X=x\right]$.

Diperoleh nilai MSE dari estimator Nadaraya-Watson yaitu:

$$
\begin{aligned}
\operatorname{MSE}(\hat{r}(x)) & =(n h)^{-1} \frac{\sigma^{2}(x)}{f(x)}\|K\|_{2}^{2}+ \\
& \frac{h^{4}}{4}\left(r^{\prime \prime}(x)+2 \frac{r^{\prime}(x) f^{\prime}(x)}{f(x)}\right)^{2} \mu_{2}^{2}(K)+ \\
& o((n h))^{-1}+o\left(h^{4}\right), h \rightarrow 0, n h \rightarrow \infty
\end{aligned}
$$

\section{Estimasi Densitas Kernel Dengan}

\section{Kernel Berorde Tinggi}

Teorema 2.1 (Roussas, 1973) Bila $X$ variabel random tak negatif maka $P[X \geq a] \leq \frac{E(X)}{a}$.

Teorema 2.2 (Roussas, 1973) Bila $X$ variabel random dengan $E(X)=\mu$, $\operatorname{var}(X)$ $=\sigma^{2}$ maka $P[|X-\mu|>\varepsilon] \leq \sigma^{2} / \varepsilon^{2}, \varepsilon>0$.

Definisi 2.1 (Roussas, 1973) Barisan variabel random $\left\{X_{n}\right\}$ dikatakan konvergen ke $X$ (dalam probabilitas), dinotasikan $X_{n} \stackrel{P}{\longrightarrow} X$, jika untuk setiap $\quad \varepsilon>0, P\left[\left|X_{n}-X\right| \geq \varepsilon\right] \rightarrow 0$ untuk $n \rightarrow \infty$.

Lemma 2.1 (Roussas, 1973) Jika $X_{n} \stackrel{P}{\longrightarrow} X$ dan $Y_{n} \stackrel{P}{\longrightarrow} Y \quad$ maka
$\frac{X_{n}}{Y_{n}} \stackrel{P}{\longrightarrow} \frac{X}{Y}$

dimana

$P\left(Y_{n}=0\right)=P(Y=0)=0$.

Suatu kernel dikatakan berorde $v$ jika memenuhi syarat sebagai berikut: $K(x) \geq 0$, untuk semua nilai $x \in \square$, $\int K(x) d x=1$, $\int x^{j} K(x) d x=\gamma_{j}\left\{\begin{array}{l}=0, j=1, \ldots, v-1 \\ \neq 0, j=v\end{array}\right.$.

Teorema 2.1.1 (Hardle, 1991) Andaikan kernel $K$ berorde tinggi, $\hat{f}_{h}(x)$ merupakan estimator dari fungsi densitas $f$ yang mempunyai turunan kontinu terbatas $p$ dan $v$ adalah orde kernel, maka bias dari fungsi $f$ tersebut adalah $\gamma^{t} \frac{h^{t}}{t !} f^{(t)}(x)+o\left(h^{t}\right), \quad h \rightarrow 0 \quad$ dimana $t$ $=\min \{p, v\}$ dengan asumsi sebagai berikut:

(i) Fungsi $f$ bersifat kontinu dan terintegralkan secara kuadrat

(ii) Bandwidth $h$ memenuhi asumsi $\lim _{n \rightarrow \infty} h=0$ dan $\lim _{n \rightarrow \infty} n h=\infty$

Fungsi kernel $K$ merupakan fungsi kepadatan probabilitas yang terbatas dan simetri di sekitar daerah aslinya.

3. Sifat Asimtotik Estimator Nadaraya Watson Dengan Kernel Orde Tak Hingga

\subsection{Kernel orde tak hingga}

Sifat Asimtotik ... (Maria Suci Apriani) 
Menurut Berg (2008) fungsi Kernel dikatakan mempunyai orde $v$ jika memenuhi: $\int_{R}|x|^{v}|K(x)| d x<\infty$ dan $\int_{-\infty}^{\infty} x^{i} K(x) d x=0, \quad i=1,2, \ldots, v-1$.

Definisi 3.1.1 (Berg, 2008). $K(x)$ dikatakan berorde tak hingga jika memenuhi:

$$
\int_{-\infty}^{\infty} x^{i} K(x) d x=0, \quad i=1,2, \ldots
$$

\section{Definisi 3.1.2 (McMurry dan Politis,} 2003). Sebuah flat-top Kernel $K$ dengan orde tak hingga secara umum dibentuk melalui Transformasi Fourier $\lambda$, yaitu untuk nilai tetap $c>0$

$$
\lambda(s)= \begin{cases}1 & j i k a|s| \leq c \\ g(|s|) & j i k a|s|>c\end{cases}
$$

Dimana fungsi $g$ dipilih sehingga membuat $\lambda(s), \lambda^{2}(s)$ dan $s \lambda(s)$ dapat diintegralkan. Flat-top Kernel diberikan sebagai berikut:

$$
K(x)=\frac{1}{2 \pi} \int_{-\infty}^{\infty} \lambda(s) e^{-i s x} d s
$$

\subsection{Sifat asimtotik estimator Nadaraya}

Watson dengan kernel orde tak hingga

Akan diuji perilaku dari estimator Nadaraya-Watson dengan kernel orde tak hingga untuk $n$ pengamatan pasangan data $\left(X_{1}, Y_{1}\right),\left(X_{2}, Y_{2}\right), \ldots,\left(X_{n}, Y_{n}\right)$ yang iid dengan densitas $f$.

Asumsi 3.2.1 Ketika $n \rightarrow \infty$, bandwidth $h \rightarrow 0$ dan $n h \rightarrow \infty$.

Asumsi 3.2.2 $\varepsilon_{i}$ adalah random error dengan asumsi independen, $E\left(\varepsilon_{i} \mid X_{i}=x\right)=0$ dan $E\left(\varepsilon^{2}{ }_{i} \mid X_{i}=x\right)=\sigma^{2}$

\section{Asumsi}

$\left(X_{1}, Y_{1}\right),\left(X_{2}, Y_{2}\right), \ldots,\left(X_{n}, Y_{n}\right)$

berdistribusi identik dan independen dengan densitas $f$.

Lemma 3.2.1 Jika $x$ berada dalam interval terbuka dimana $f(x)$ mempunyai turunan kontinu terbatas $p$ dan $r(x)$ mempunyai turunan kontinu terbatas q, maka berdasarkan asumsi

\subsection{1 dan 3.2.2:}

a. $E[\hat{f}(x)]-f(x)=o\left(h^{p}\right)$

b. $E[\hat{g}(x)]-g(x)=o\left(h^{k}\right), \quad$ dimana $k=\min \{p, q\}$.

Bukti:

a. Bias penyebut estimator NadarayaWatson dengan kernel orde tak hingga Diketahui,

$$
\begin{aligned}
E\left(\hat{f}_{h}(x)\right)= & \int_{-\infty}^{\infty} K(s) f(x+s h) d s \\
= & f(x)+ \\
& \int_{-\infty}^{\infty} \frac{f^{p+1}(x+\xi)(s h)^{p+1}}{(p+1) !} K(s) d s
\end{aligned}
$$




$$
\begin{aligned}
\operatorname{bias}[\hat{f}(x)]= & f(x)+ \\
& \int_{-\infty}^{\infty} \frac{f^{p+1}(x+\xi)(s h)^{p+1}}{(p+1) !} K(s) d s- \\
& f(x) .
\end{aligned}
$$

Berdasarkan asumsi 3.2.1 maka $\operatorname{bias}[\hat{f}(x)]=o\left(h^{p}\right)$.

b. Bias pembilang estimator NadarayaWatson dengan kernel orde tak hingga

$$
\begin{aligned}
& E[\hat{g}(x)]=E\left[\frac{1}{n} \sum_{i=1}^{n} K_{h}\left(x-X_{i}\right) Y_{i}\right] \\
& =\int_{-\infty}^{\infty} K_{h}(x-u) f(u) \int_{-\infty}^{\infty} y f(y \mid u) d y d u \\
& =\int_{-\infty}^{\infty} K_{h}(x-u) f(u) r(u) d u \\
& =\int_{-\infty}^{\infty} r(u) f(u) K_{h}(x-u) d u
\end{aligned}
$$

Andaikan $(r f)$ terturunkan kontinu pada selang tertutup $[\infty,-\infty]$ dan $(r f)$ mempunyai turunan kontinu terbatas $k$ pada interval terbuka $(\infty,-\infty)$ yang memuat nilai $x$ dengan $k=\min \{p, q\}$ dan andaikan $(r f)$ merupakan fungsi mulus pada semua bilangan real $\mathbb{R}$, maka:

$$
\begin{aligned}
E(\hat{g}(x))= & \int_{-\infty}^{\infty}[(r f)(x)+ \\
& v h(r f)^{\prime}(x)+\cdots+ \\
& \frac{(v h)^{k+1}}{(k+1) !}(r f)^{(k+1)} \\
& (x+\xi)] K(v) d v .
\end{aligned}
$$

Ketika $K$ terintegralkan ke satu, semua momennya adalah nol dan ketika

$$
\begin{aligned}
& g(x)=r(x) f(x) \quad \text { maka } \\
& E(\hat{g}(x))=g(x)+ \\
& \int_{-\infty}^{\infty} \frac{(v h)^{k+1}(k f)^{(k+1)}(x+\xi) K(v) d v}{(k+1) !}
\end{aligned}
$$

Berdasarkan asumsi 3.2.1 maka

$$
E(\hat{g}(x))-g(x)=o\left(h^{k}\right) \text {. }
$$

Asumsi 3.2.4 Titik $x$ merupakan titik kontinu dari $\sigma^{2}(x), f(x)>C$ untuk $C>$ $O$ dan fungsi $r$ serta fungsi $f$ masingmasing terdiferensial di sekitar $x$.

Lemma 3.2.2 Jika $x$ berada dalam interval terbuka dimana $f(x)$ mempunyai turunan kontinu terbatas $p$ dan $r(x)$ mempunyai turunan kontinu terbatas q, berdasarkan asumsi 3.2.1 3.2.4 maka: $\operatorname{var}[\hat{f}(x)]=\frac{f(x)}{n h} \int_{-\infty}^{\infty} K^{2}(z) d z+$

$$
o\left(\frac{1}{n h}\right)+O\left(\frac{1}{n}\right)
$$

b.

$$
\begin{gathered}
\operatorname{var}[\hat{g}(x)]=\frac{\left(r^{2}(x)+\sigma^{2}(x)\right) f(x)}{n h} \int_{-\infty}^{\infty} K^{2}(z) d z+ \\
o\left(\frac{1}{n h}\right)+O\left(\frac{1}{n}\right)
\end{gathered}
$$

Bukti:

$$
\begin{aligned}
& \text { a. } \quad \operatorname{var}(\hat{f}(x))=\operatorname{var}\left[\frac{1}{n} \sum_{i=1}^{n} K_{h}\left(x-X_{i}\right)\right] \\
& =n^{-1}\left(E\left[K_{h}^{2}(x-u)\right]-\left(E\left[K_{h}(x-u)\right]\right)^{2}\right)
\end{aligned}
$$

Dimana, 


$$
\begin{aligned}
& E\left[K_{h}^{2}(x-u)\right]=\int_{-\infty}^{\infty} K_{h}^{2}(x-u) f(u) d u \\
&=\frac{1}{h} \int_{-\infty}^{\infty} K^{2}(s) f(x+s h) d s \\
&=\frac{1}{h}\left\{\int _ { - \infty } ^ { \infty } K ^ { 2 } ( s ) \left[f(x)+s h f^{\prime}(x)+\right.\right. \\
& \frac{h^{2} s^{2}}{2} f^{\prime \prime}(x)+\cdots+\frac{h^{p} s^{p}}{p !} f^{p}(x)+ \\
&\left.\left.\frac{h^{p+1} s^{p+1}}{(p+1) !} f^{p+1}(x+\xi)\right] d s\right\} .
\end{aligned}
$$$$
=\frac{1}{n}\left[E\left[K^{2}{ }_{h}(x-u) y^{2}\right]-E\left[K_{h}(x-u) y\right]^{2}\right]
$$

Dimana,

$$
\begin{aligned}
& E\left[K^{2}{ }_{h}(x-u) y^{2}\right]=\int_{-\infty}^{\infty} \int_{-\infty}^{\infty} K^{2}{ }_{h}(x-u) y^{2} f(u, y) d u d y \\
& =\int_{-\infty}^{\infty} K^{2}{ }_{h}(x-u) f(u) E\left(\left(r(u)+\varepsilon_{i}\right)^{2} \mid X=u\right) d u \\
& =\int_{-\infty}^{\infty} K_{h}^{2}(x-u) f(u)\left(r^{2}(u)+\sigma^{2}(u)\right) d u .
\end{aligned}
$$

Sehingga:

$$
\begin{aligned}
& \operatorname{var}(\hat{f}(x))=n^{-1}\left[E\left[K_{h}^{2}(x-u)\right]-\right. \\
& \left.\left(E\left[K_{h}(x-u)\right]\right)^{2}\right] \\
& =\frac{1}{n h} \int_{-\infty}^{\infty} K^{2}(s) f(x) d s+ \\
& \frac{1}{n h}\left(\int _ { - \infty } ^ { \infty } K ^ { 2 } ( s ) \left[s h f^{\prime}(x)+\right.\right. \\
& \frac{h^{2} s^{2}}{2} f^{\prime \prime}(x)+\cdots+\frac{h^{p} s^{p}}{p !} f^{p}(x)+ \\
& \left.\left.\frac{h^{p+1} s^{p+1}}{(p+1) !} f^{p+1}(x+\xi)\right] d s\right)- \\
& \frac{1}{n}\left(f(x)+o\left(h^{p}\right)\right)^{2}
\end{aligned}
$$

Sehingga didapatkan:

$$
\begin{aligned}
\operatorname{var}(\hat{g}(x))= & \frac{1}{n}\left[\int_{-\infty}^{\infty} K^{2}{ }_{h}(x-u) f(u)\right. \\
& \left(r^{2}(u)+\sigma^{2}(u)\right) d u- \\
& \left.\left(g(x)+o\left(h^{k}\right)\right)^{2}\right] \\
= & \frac{1}{n h} \int_{-\infty}^{\infty} K^{2}(s) f(x+s h) \\
& \left(r^{2}(x+s h)+\sigma^{2}(x+s h)\right) d s \\
& -\frac{1}{n}\left(g(x)+o\left(h^{k}\right)\right)^{2} .
\end{aligned}
$$

Berdasarkan asumsi 3.2.1 dan dengan

Berdasar asumsi $\quad 3.2 .1 \quad$ maka

$$
\begin{aligned}
& \operatorname{var}(\hat{f}(x))= \\
& \frac{1}{n h} \int_{-\infty}^{\infty} K^{2}(s) f(x) d s+o\left(\frac{1}{n h}\right)+O\left(\frac{1}{n}\right)
\end{aligned}
$$
deret Taylor maka:

$$
\begin{aligned}
\operatorname{var}(\hat{g}(x))= & \frac{\left(r^{2}(x)+\sigma^{2}(x)\right) f(x)}{n h} \\
& \int_{-\infty}^{\infty} K^{2}(s) d s+o\left(\frac{1}{n h}\right)+O\left(\frac{1}{n}\right) .
\end{aligned}
$$

b. $\quad \operatorname{var}(\hat{g}(x))=\operatorname{var}\left[\frac{1}{n} \sum_{i=1}^{n} K_{h}\left(x-X_{i}\right) Y_{i}\right]$

Lemma 3.2.3 Berdasarkan asumsi 3.2.1

-3.2.4 serta lemma 3.2.1 dan 3.2.2 maka:

a. $\hat{f}(x) \stackrel{p}{\longrightarrow} f(x)$ 
b. $\hat{g}(x) \stackrel{p}{\longrightarrow} g(x)$

Bukti:

a. Akan dibuktikan $\hat{f}(x)$ konvergen dalam probabilitas ke $f(x)$.

Dengan menggunakan ketaksamaan Chebychev, maka:

$P\left(\left|\hat{f}(x)-\left(f(x)+o\left(h^{p}\right)\right)\right|>\varepsilon\right) \leq$ $\frac{\frac{1}{n h} \int_{-\infty}^{\infty} K^{2}(s) f(x) d s+o\left(\frac{1}{n h}\right)+O\left(\frac{1}{n}\right)}{\varepsilon^{2}}$.

Ketika $\quad n \rightarrow \infty \quad$ maka $\operatorname{var}(\hat{f}(x)) \rightarrow 0, \quad$ hal ini menunjukkan bahwa $\hat{f}(x) \stackrel{p}{\longrightarrow} f(x)$.

b. Akan dibuktikan $\hat{g}(x)$ konvergen dalam probabilitas ke $g(x)$.

Dengan menggunakan ketaksamaan Chebychev dan berdasarkan lemma 3.2.1 serta lemma 3.2.2 maka:

$$
\begin{aligned}
& P\left(\mid \hat{g}(x)-\left(g(x)+o\left(h^{k}\right) \mid>\varepsilon\right) \leq\right. \\
& {\left[\frac{\left(r^{2}(x)+\sigma^{2}(x)\right) f(x)}{n h} \int_{-\infty}^{\infty} K^{2}(z) d z+\right.} \\
& \left.o\left(\frac{1}{n h}\right)+O\left(\frac{1}{n}\right)\right] 1 / \varepsilon^{2} .
\end{aligned}
$$

Ketika $n \rightarrow \infty$ maka $\operatorname{var}(\hat{g}(x)) \rightarrow 0$ , hal ini menunjukkan bahwa $\hat{g}(x) \stackrel{p}{\longrightarrow} g(x)$.
Berdasarkan Lemma 2.1 maka $\hat{r}(x)=\frac{\hat{g}(x)}{\hat{f}(x)} \stackrel{p}{\longrightarrow} \frac{g(x)}{f(x)}=r(x)$.

Terbukti $\hat{r}(x)$ merupakan estimator yang konsisten secara asimtotik pada kurva regresi $r(x)$.

Teorema 3.2.1 Jika $x$ berada dalam interval terbuka dimana $f(x)$ mempunyai turunan kontinu terbatas $p$ dan $r(x)$ mempunyai turunan kontinu terbatas q maka berdasarkan asumsi 3.2.1-3.2.4,

$$
\begin{aligned}
& \sqrt{n h}\left(\hat{r}(x)-r(x)+o\left(h^{q}\right)\right) \stackrel{D}{\longrightarrow} \\
& N\left(0, \frac{\sigma^{2}(x)}{f(x)} \int_{-\infty}^{\infty} K^{2}(z) d z\right) .
\end{aligned}
$$

Bukti:

$$
\begin{aligned}
Y_{i}=r\left(X_{i}\right)+\varepsilon_{i} & \\
\frac{1}{n} \sum_{i=1}^{n} K_{h}\left(x-X_{i}\right) Y_{i}= & \frac{1}{n} \sum_{i=1}^{n} K_{h}\left(x-X_{i}\right) r(x)+ \\
& \frac{1}{n} \sum_{i=1}^{n} K_{h}\left(x-X_{i}\right) \\
& \left(r\left(X_{i}\right)-r(x)\right)+ \\
& \frac{1}{n} \sum_{i=1}^{n} K_{h}\left(x-X_{i}\right) \varepsilon_{i} .
\end{aligned}
$$

Dapat ditulis:

$$
\hat{r}(x)=r(x)+\frac{\hat{a}_{1}(x)}{\hat{f}(x)}+\frac{\hat{a}_{2}(x)}{\hat{f}(x)} .
$$

1. Akan dibuktikan $\hat{a}_{2}(x)$ berdistribusi normal secara asimtotik. 
Andaikan $\quad b_{i}(x)=\frac{K\left(\frac{x-X_{i}}{h}\right) \varepsilon_{i}}{\sqrt{h}}$, maka $\sqrt{n h} \hat{a}_{2}(x)=\frac{1}{\sqrt{n}} \sum_{i=1}^{n} b_{i}(x)$. Kita dapatkan $E\left(b_{i}(x)\right)=0$, $\operatorname{var}\left(b_{i}(x)\right)=\int K^{2}(s) d s \sigma^{2}(x) f(x)$.

Akan dibuktikan bahwa $b_{i}(x)$ memenuhi syarat Liapunov.

$$
\begin{aligned}
& \sum_{i=1}^{n} E\left[\left|\frac{1}{\sqrt{n}} b_{i}(x)\right|^{2+\delta}\right]= \\
& \sum_{i=1}^{n} E\left[\left|\frac{1}{\sqrt{n h}} K\left(\frac{x-X_{i}}{h}\right) \varepsilon_{i}\right|^{2+\delta}\right] \\
& =O\left(\frac{1}{\sqrt{n h}}\right)^{\delta} .
\end{aligned}
$$

Ketika $\quad n \rightarrow \infty$ diperoleh $\sum_{i=1}^{n} E\left[\left|\frac{1}{\sqrt{n}} b_{i}(x)\right|^{2+\delta}\right] \rightarrow 0$.

Sehingga

$$
\sqrt{n h} \hat{a}_{2}(x) \stackrel{D}{\longrightarrow} N\left(0, \int K^{2}(s) d s \sigma^{2}(x) f(x)\right)
$$

2. Akan dibuktikan

$$
\hat{a}_{1}(x)-f(x) o\left(h^{q}\right) \stackrel{P}{\longrightarrow} 0 .
$$

a. Nilai ekspektasi dari $\hat{a}_{1}(x)$.

$$
\begin{aligned}
E\left[\hat{a}_{1}(x)\right]= & \frac{1}{n h} E\left[\sum_{i=1}^{n} K\left(\frac{x-X_{i}}{h}\right)\right. \\
& \left.\left(r\left(X_{i}\right)-r(x)\right)\right]
\end{aligned}
$$

$$
\begin{aligned}
& =o\left(h^{k}\right)+f(x) o\left(h^{q}\right) \\
& \approx f(x) o\left(h^{q}\right)
\end{aligned}
$$

b. Nilai variansi dari $\hat{a}_{1}(x)$.

$$
\begin{aligned}
& \operatorname{var}\left(\hat{a}_{1}(x)\right)=\operatorname{var}\left[\frac{1}{n h} \sum_{i=1}^{n} K\left(\frac{x-X_{i}}{h}\right)\left(r\left(X_{i}\right)-r(x)\right)\right] \\
& =\frac{1}{n h}\left[\begin{array}{l}
\int_{-\infty}^{\infty} K^{2}(s)(r(x+s h)-r(x))^{2} \\
f(x+s h) d s-\left[f(x) o\left(h^{q}\right)\right]^{2}
\end{array}\right] \\
& =O\left(\frac{1}{n h}\right)
\end{aligned}
$$

Menggunakan ketaksamaan Chebychev dan ketika $n \rightarrow \infty$ maka:

$$
\operatorname{var}\left(\hat{a}_{1}(x)\right) \rightarrow 0, \quad \text { sehingga }
$$

$\hat{a}_{1}(x)-f(x) o\left(h^{q}\right) \stackrel{P}{\longrightarrow} 0 \quad$ akibatnya $\sqrt{n h}\left(\hat{a}_{1}(x)-f(x) o\left(h^{q}\right)\right) \stackrel{P}{\longrightarrow} 0$. Telah dibuktikan bahwa $\hat{f}(x) \stackrel{p}{\longrightarrow} f(x)$, sehingga:

$$
\sqrt{n h}\left(\frac{\hat{a}_{1}(x)}{\hat{f}(x)}-o\left(h^{q}\right)\right) \stackrel{P}{\longrightarrow} 0
$$

3. Membuktikan estimator $\hat{r}(x)$ berdistribusi normal secara asimtotik.

$$
\begin{aligned}
& \sqrt{n h}\left(\hat{r}(x)-r(x)-o\left(h^{q}\right)\right)= \\
& \sqrt{n h}\left(\frac{\hat{a}_{1}(x)}{\hat{f}(x)}-o\left(h^{q}\right)\right)+\frac{\sqrt{n h} \hat{a}_{2}(x)}{\hat{f}(x)}
\end{aligned}
$$

Berdasarkan poin 1 dan 2 maka: 


$$
\begin{aligned}
& \sqrt{n h}\left(\hat{r}(x)-r(x)-o\left(h^{q}\right)\right) \stackrel{D}{\longrightarrow} \\
& N\left(0, \frac{\int K^{2}(s) d s \sigma^{2}(x)}{f(x)}\right)
\end{aligned}
$$

Terbukti bahwa estimator $\hat{r}(x)$ berdistribusi normal secara asimtotik.

\section{Kesimpulan}

Secara asimtotik estimator Nadaraya Watson dengan Kernel orde tak hingga memiliki sifat konsisten secara probabilitas dan normal. Sifat kenormalan dari estimator tersebut dengan menggunakan kernel orde tak hingga memiliki rata-rata nol dan variansi

$$
\frac{\int K^{2}(s) d s \sigma^{2}(x)}{f(x)}
$$

\section{Pustaka}

Berg, Arthur. 2008. Nonparametric Function Estimation with InfiniteOrde Kernels. Department of Statistics, University of Florida.

Hardle, Wolfgang. 1991. Smoothing Techniques With Implementation in S. Springer-Verlag, New York.

McMurry, T.L and Politis, D.N. 2003. Nonparametric Regression with Infinite Orde Flat-Top Kernel, tersedia di www.math.ucsd.edu/ politis/PAP ER/McMurryPolitis04.pdf, diakses pada tanggal 13 Februari 2013.

Roussas, G.G. 1973. A First Course in Mathematical Statistics. AddisonWesley Publishing Company, Inc. Taiwan. 
